\title{
The Effects of Concurrent Training during Cardiac Rehabilitation on Plasma MMP-9 and TIMP-1 Levels in Myocardial Ischemic Patients
}

\author{
Mostafa Dastani ${ }^{1 *}$, Bahareh Radfar ${ }^{2}$, Artemis Naghibzadeh ${ }^{3}$, Farida Sadeghi Fazel ${ }^{3}$, Seyed Emad \\ Alavinia $^{3}$, Mohamad Motevallii, ${ }^{4,5}$ and Amir Rashidlamir ${ }^{5}$ \\ ${ }^{1}$ Department of Cardiology, Mashhad University of Medical Sciences, Mashhad, Iran \\ ${ }^{2}$ Department of Physical Education, Faculty of Humanities and Social Sciences, Yazd University, Yazd, Iran \\ ${ }^{3}$ Faculty of Physical Education and Sport Sciences, Islamic Azad University of Neyshabur, Neyshabur, Iran \\ ${ }^{4}$ Department of Sport Science, Leopold-Franzens University of Innsbruck, Innsbruck, Austria \\ ${ }^{5}$ Department of Exercise Physiology, Faculty of Sport Sciences, Ferdowsi University of Mashhad, Mashhad, Iran \\ *Corresponding author: Mostafa Dastani, Department of Cardiology, Mashhad University of Medical Sciences, Mashhad, Iran
}

\section{ARTICLE INFO}

Received: 亚 August 24, 2021

Published: 慧 September 03, 2021

Citation: Mostafa Dastani, Bahareh Radfar, Artemis Naghibzadeh, Farida Sadeghi Faze, Seyed Emad Alavinia, et al., The Effects of Concurrent Training during Cardiac Rehabilitation on Plasma MMP-9 and TIMP-1 Levels in Myocardial Ischemic Patients. Biomed J Sci \& Tech Res 38(4)2021. BJSTR. MS.ID.006173.

Abbreviations: MMPs: Matrix Metalloproteinase Enzymes; TIMP-1: Tissue Inhibitor of Metalloproteinase-1; 1RM: One Repetition Maximum; EG: Experimental Group; CG: Control Group; MHR: Maximum Heart Rate; RPE: Borg Rating of Perceived Exertion; ELISA: Enzyme-Linked Immunosorbent Assay; MCP-1: Monocyte Chemoattractant Protein-1; Ox-LDL: Oxidized Low-Density Lipoprotein

\section{ABSTRACT}

Background: Matrix metalloproteinase enzymes (MMPs) play a destructive role in atherosclerosis resulting in occurrence of cardiac ischemia. The aim of this study was to investigate the effect of concurrent training during cardiac rehabilitation on plasma levels of MMP-9 and tissue inhibitor of metalloproteinase-1 (TIMP-1) in patients with myocardial ischemia.

Methods: Sixteen cardiac ischemic patients who were in the process of clinical cardiac rehabilitation were randomly assigned to experimental $(n=8)$ or control $(n=$ 8) groups. The patients in experimental group performed concurrent exercise for eight weeks (one hour / three session per week) with the intensity of $40-60 \%$ of one repetition maximum and $60-80 \%$ maximum heart rate. Pre- and post-intervention blood samples were taken to assess plasma MMP-9 and TIMP-1 concentrations using ELISA method.

Findings: Independent T-test showed the patients in experimental group had a significant decrease $(t=2.431 ; p=0.029)$ in MMP-9 plasma concentration and a significant increase $(t=3.202 ; p=0.006)$ in plasma levels of TIMP-1 compared to the control group. A significant within-group reduction in MMP-9 levels $(\mathrm{t}=0.695 ; \mathrm{P}=$ $0.008)$ and a significant within-group increase in TIMP-1 levels $(t=3.964 ; \mathrm{P}=0.005)$ were observed in the experimental group, while the pre- to post-value changes in MMP9 and TIMP-1 levels in the control group were not statistically significant.

Conclusion: It seems that the favorable changes in MMP-9 and TIMP-1 following eight weeks of physical exercise prevent the progression of atherosclerosis and recurrence of cardiac ischemia during the process of cardiac rehabilitation.

Keywords: Matrix Metallopeptidases, Tissue Inhibitor of Metalloproteinase, Exercise Training, Cardiac Rehabilitation, Cardiac Ischemia 


\section{Introduction}

Cardiac ischemia is considered as one of the deadliest heart diseases. According to the reports from American Heart Association, one third of cardiac patients suffer from ischemic heart ailment [1]. Several genomic and environmental factors including but not limited to family history, gender, age, smoking, hypertension, diabetes, hyperlipidemia, and lack of physical activity can potentially contribute to atherosclerosis and subsequently ischemic heart disease [2,3]. Atherosclerosis, a chronic inflammatory disease, is characterized as the accumulation of fat and other substances on the inner surface of artery walls [4]. In the atherosclerotic situation, due to the deficiency of blood flow in the coronary arteries, an acute blockage occurs which is defined as a heart attack [5]. With the progression of atherosclerosis, the smooth muscle cells move toward the intima in response to growth factors released by active macrophages and endothelial cells. Active macrophages weaken the extracellular matrix and support the platelet fibrosis by producing matrix metalloproteinases (MMPs) suggesting a prominent role in the process of atherosclerosis and rupturing the artery walls [6]. Matrix metalloproteinases (MMPs), which are a large group of protease enzymes, are responsible for the breaking down of extracellular matrix.

To date, 26 members of MMP family have been identified, and among them, gelatinase-B (or MMP-9) has a higher activity than other members [7] with a well-documented potential in developing cardiovascular disease in human populations [8]. Based on the evidence, myocardium expression of MMP-9 is increased in the patients with coronary artery disease $[9,10]$. However, due to the damaging role of MMPs, their activity is strongly regulating by four tissue inhibitors (TIMPs) namely TIMP-1, TIMP-2, TIMP-3, and TIMP-4 which can control the detrimental activities of MPPs in vertebrates [11]. Among the TIMP family, it has been reported that TIMP-1 is a potent inhibitor of many MMPs including MMP-9 [12] and play a key role in the structure and function of myocardium ae well as the control of extracellular matrix proteolysis in cardiovascular system [12]. Recurrence of cardiac ischemia and its unfavorable consequences are a major concern among cardiac ischemic patients as well as cardiovascular specialists [8]. Nowadays, with developing clinical centers, medical strategies, and patients' knowledge in diagnosing early signs of heart disease the rate of mortality has been clearly decreased; however, some of these patients with the acute symptoms have been hospitalized for the second and third time and some of them still may have died [13].

In addition to pharmacological treatments and implementing medical interventions on coronary arteries, cardiac rehabilitation program has been well-documented in controlling the risk factors, improving life quality, and reducing the mortality rate [14]. The cardiac rehabilitation program is designed to limit the physiological and psychological consequences of cardiovascular disease, reduce the risk of sudden death or stroke, control cardiac symptoms, and decline the atherosclerosis [15]. Physical exercise is frequently used as a non-pharmacological and supplemental method in the process of medical treatment for a variety of reasons [16]. As an incentive method, physical exercise can be used during cardiac rehabilitation programs due to its low cost and attractiveness [17]. The type of physical exercise used in cardiac rehabilitation programs is generally aerobic in forms of walking, running, or cycling [18]. However, it has been recently reported that a welldesigned resistance training program accompanied by aerobic exercise can be considered as a helpful strategy in rehabilitation programs. In this regard, the American Heart Association has also recommended that resistance training be performed twice a week in cardiac rehabilitation programs [18].

According to the recent scientific reports, the implementation of physical exercise in rehabilitation programs can lead to effective outcomes on some clinical indicators involved in the occurrence of atherosclerosis [19]. In a study examining the effect of cardiac rehabilitation on the atherosclerosis biomarkers in patients with cardiac ischemia, the authors reported that performing physical exercise during cardiac rehabilitation programs will prevent reoccurrence of cardiac ischemia [20]. However, due to the increasing prevalence of ischemic heart disease [1] and considering the importance role of MMPs particularly MMP-9 in the formation and stabilization of atherosclerotic plaque, few studies have yet examined the effects of a well-planed physical exercise during cardiac rehabilitation on the levels of metalloproteinases and their tissue inhibitors in patients with cardiac ischemia [21,22]. Therefore, the need for a comprehensive investigation in this promising area is clearly evident and the present study is designed to examine this imperative issue.

\section{Materials and Methods}

The present study is an experimental controlled clinical trial. Sixteen cardiovascular patients with atherosclerotic symptoms who were referred to a cardiac hospital in Mashhad, Iran were selected and randomly assigned to an experimental $(n=8)$ or control $(n=8)$ group. Scheme of the study design is shown in Figure 1. Before the commencement of the study, patients in both groups were monitored by a cardiologist and were reported to have symptoms of coronary heart disease. For each patient, a file including demographic information, history, clinical cardiac reports, and anthropometric data was provided. At the beginning of the rehabilitation program, an initial assessment of cardiac function was conducted using echocardiographic devices. During the study, participants in both groups followed the prescribed medications and diets (with a particular amount of calorie and macronutrients per kilogram of body weight for all participants) which were provided by a dietitian 
and a cardiologist. The patients in experimental group completed an eight-week moderate concurrent exercise program with 40-60\% of their one maximum repetition.

According to the American Heart Association guidelines, the patient's exercise intensity was monitored to be between 60 and $80 \%$ of their maximum heart rate [23] using the individualized electrocardiograms connecting to each participant during exercise sessions. Moreover, Borg Rating of Perceived Exertion (RPE) scale was also used to a further control of the exercise intensity, and the patients in experimental group were asked to keep their activity intensity between level 11 (relatively light) and level 13 (somewhat difficult) [24]. The concurrent training protocol is shown in Table 1. Before the commencement of training sessions, participants in experimental group were informed and introduced to devices and exercises in one session and then practiced three sessions per week for eight weeks. Each training session lasted about one hour performing mixed aerobic and anaerobic exercises using treadmills, bikes, ergometers, physio balls, and light weights. At the starting of each raining sessions 5 minutes of warm-up, 5 minutes of walking on treadmill, 5 minutes of bike pedaling, and 5 minutes of upperbody ergometer pedaling were done with three minutes of inactive rest between each aerobic exercise.

Table 1: Concurrent training protocol.

\begin{tabular}{|c|c|c|c|c|c|c|c|}
\hline \multirow{2}{*}{ Week } & \multirow{2}{*}{$\begin{array}{l}\text { Training } \\
\text { Sessions }\end{array}$} & \multicolumn{4}{|c|}{ Resistance Training } & \multicolumn{2}{|c|}{ Endurance Training } \\
\hline & & Sets & $\begin{array}{l}\text { Rest Interval } \\
\text { (min) }\end{array}$ & Repetition & $\begin{array}{l}\text { Intensity } \\
\text { (\%1RM) }\end{array}$ & Volume (min) & $\begin{array}{l}\text { Intensity } \\
\text { (\%MHR) }\end{array}$ \\
\hline 1 & 3 & 3 & 2 & 10 & 40 & 15 & $60-65$ \\
\hline 2 & 3 & 3 & 2 & $10-11$ & 40 & 15 & $60-65$ \\
\hline 3 & 3 & 3 & 2 & $11-12$ & 40 & 15 & $65-70$ \\
\hline 4 & 3 & 3 & 2 & $11-13$ & 50 & 15 & $65-70$ \\
\hline 5 & 3 & 3 & 2 & $12-13$ & 50 & 15 & $70-75$ \\
\hline 6 & 3 & 3 & 2 & $13-14$ & 50 & 15 & $70-75$ \\
\hline 7 & 3 & 3 & 2 & $14-15$ & 60 & 15 & $75-80$ \\
\hline 8 & 3 & 3 & 2 & 15 & 60 & 15 & $75-80$ \\
\hline
\end{tabular}

Note: 1RM: One Repetition Maximum; MHR: Maximum Heart Rate.

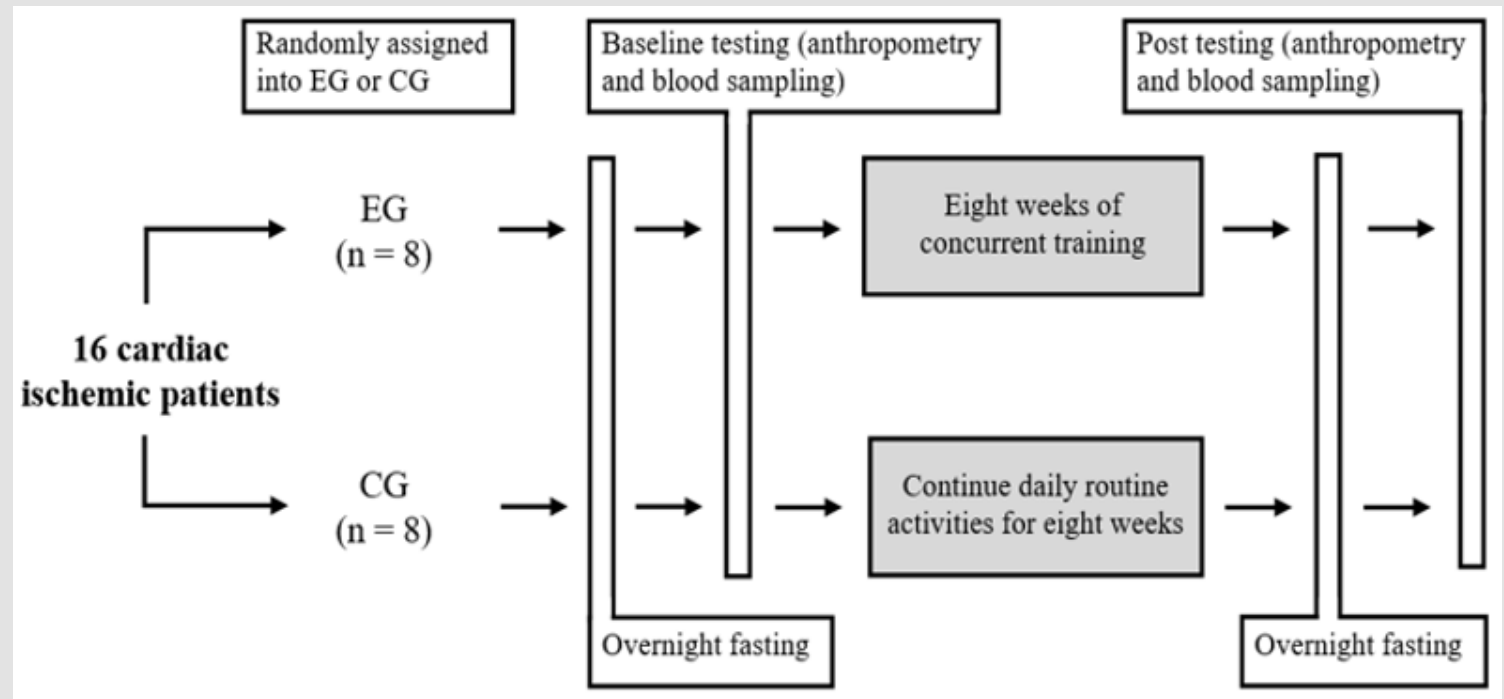

Figure 1: Schematic of the study design. EG: experimental group; CG: control group. 
After 5 minutes of rest, the individuals gradually performed the following workouts with 10 repetitions in three sets in the initial training sessions and to 15 repetitions in the advanced sessions. The workouts consisted of squat with a physio ball, shoulder flexion, shoulder abduction, elbow flexion, hip flexion, hip abduction, ankle plantar flexion, and ankle dorsi flexion. The workouts were initially performed using the patient's own body weight or limb weight, and gradually improved using therabands and light weights [25]. A careful supervision was applied during the training sessions, and the patients were constantly questioned about the amount of pressure based on the Borg scale. If the value of Borg scale was reported below 11 or above 13, the participant was asked to increase or decrease the effort, respectively.

Laboratory Methods: Fasting $10^{\mathrm{cc}}$ blood samples were obtained 48 hours before the first training session and 48 hours after the last training session from the participants' antecubital vein in both groups. Blood samples were collected in lavendertop tubes containing anti-coagulant EDTA, and then were carried to laboratory for plasma separation by $3000^{\mathrm{rpm}}$ centrifuging for 10 minutes. Subsequently, plasma samples were frozen and stored at $-80^{\circ} \mathrm{C}$ for further analysis. Plasma MMP-9 and TIMP-1 concentrations were assessed by Enzyme-Linked Immunosorbent Assay (ELISA) method using Awareness Stat Fax 2100 device according to the instructions of the ELISA kits (R\&D Systems,
Minneapolis, MN, USA).

\section{Results}

Data distribution was normal according to the results from Shapiro Wilk test. The baseline characteristics of the participants are shown in Table 2. The independent T-test showed a significant decrease in plasma MMP-9 concentration $(t=2.431 ; p=0.029)$ and a significant increase in TIMP- 1 plasma levels $(\mathrm{t}=3.202 ; \mathrm{p}=$ 0.006) following the exercise intervention in the exercise group compared to the control group. According to the correlated T-test, a significant within-group decrease in MMP-9 levels $(t=0.695 ; p=$ 0.008 ) and a significant increase in plasma TIMP-1 concentrations $(t=3.964 ; p=0.005)$ were observed in the experimental group, while within-group changes in MMP-9 and TIMP-1 levels were not significant in the control group. $(\mathrm{t}=0.21 ; \mathrm{p}=0.838$ and $\mathrm{t}=0.46 ; \mathrm{p}=$ 0.66 , respectively) (Figure 2).

Table 2: Baseline characteristics of the participants in experimental $(n=8)$ and control $(n=8)$ groups (mean $\pm S D)$.

\begin{tabular}{|c|c|c|}
\hline \multirow{2}{*}{ Age $(\mathbf{y})$} & Experimental Group & Control Group \\
\cline { 2 - 3 } & $56.25 \pm 6.21$ & $54.09 \pm 6.33$ \\
\hline Height $(\mathrm{m})$ & $1.72 \pm 3.1$ & $1.74 \pm 4.7$ \\
\hline Weight $(\mathrm{kg})$ & $72.2 \pm 8.73$ & $76.7 \pm 7.45$ \\
\hline Body Mass Index $\left(\mathrm{kg} / \mathrm{m}^{2}\right)$ & $24.7 \pm 2.94$ & $25.11 \pm 2.24$ \\
\hline
\end{tabular}
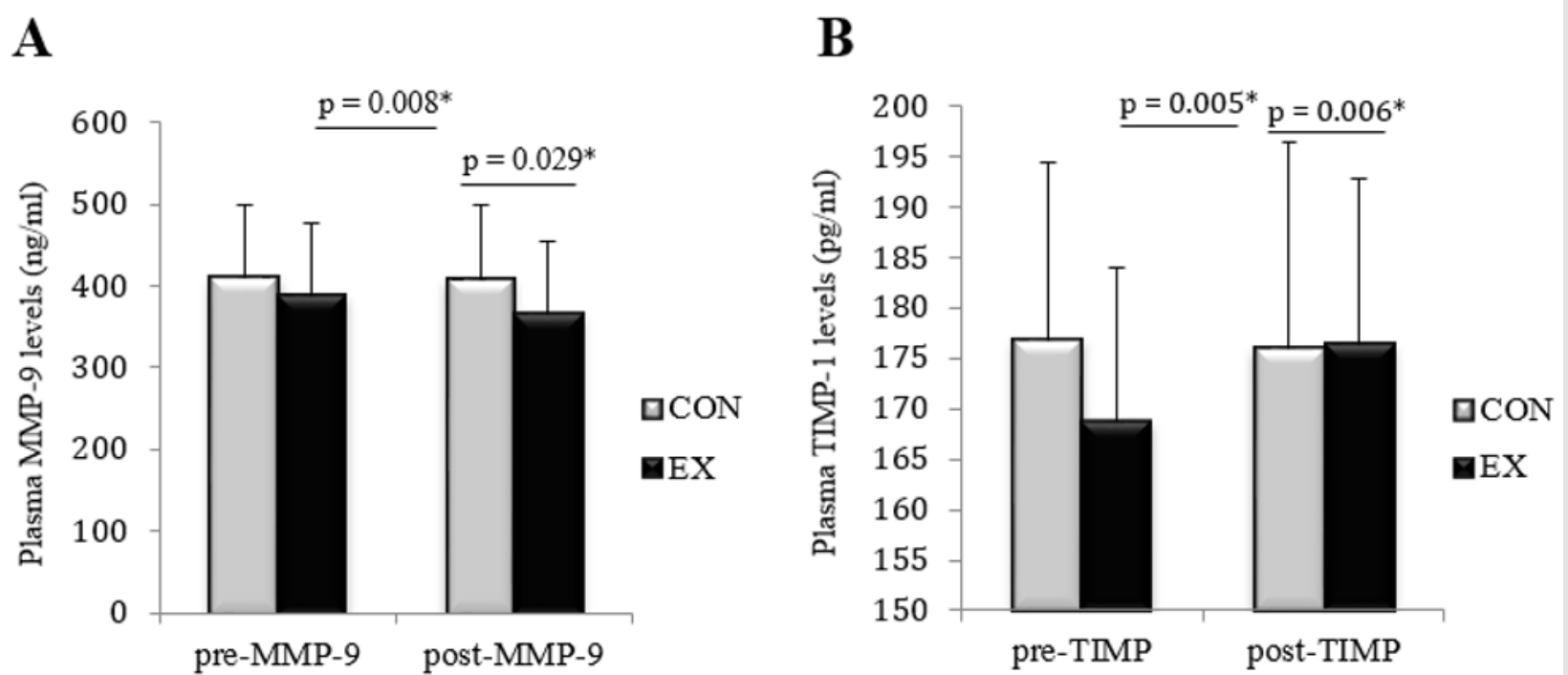

Figure 2: The average changes in plasma levels of MMP-9 (part A) and TIMP-1 (part B) between per- and post-test values in experimental (EX) and control (CON) groups.

\section{Discussion}

Present study showed that after eight weeks of moderate physical exercise during the cardiac rehabilitation period, MMP9 levels decreased and TIMP-1 levels increased, and significant within-group changes in MMP-9 and TIMP-1 levels were observed in the experimental group. To date, the investigations assessing the effects of exercise on MMPs and TIMPs levels have often been conducted in healthy, obese, or diabetic populations as well as animal models. Seydanlou and Farzanegi in 2014 reported a significant 20\% decrease in MMP-2 levels and a significant $26 \%$ 
increase in TIMP-1 levels of overweight individuals following eight weeks of Pilates training [26]. Consistent with our results, Kadoglou, et al. in 2013 found a significant increase in TIMP-1 concentrations following six weeks of treadmill running in animal models [27], similar to the results of Koskkinen's study but in humans [28]. Although the effectiveness of physical exercise on MMP-9 and TIMP-1 levels are well documented in the literature, limited studies have reported no change in the aforementioned variables following different exercise interventions. Previously, Mackey, et al. reported that a 10-kilometer road and water running has no effect on MMP-2 and MMP-9 levels in young men [29].

Moreover, according to the results of a study by Hoier, et al., eight weeks of cycling training with the intensity of $60 \%$ maximum oxygen consumption did not affect the TIMP-1 levels in healthy men [30]. However, these inconsistent findings might be justified by different training methods, varied participants' characteristics, and different sampling methods (muscle biopsy vs blood sample) used in the aforesaid investigations. Metalloproteinase matrix enzymes are believed to alter the formation of the cardiovascular matrix during natural biological processes [31]. However, in pathophysiological processes of various diseases, the expression and activity of these type of proteolytic enzymes are increased due to the increased secretion of proinflammatory cytokines leading to breakdown of several collagens and gelatinases, as well as detrimental effects on microanatomical tissue structures. As a result, exacerbation of inflammatory state and emerging of various heart and vascular diseases might occur with advancing time $[32,33]$. In a study examining the role of matrix metalloproteinase $1,2,3$, and 9 in acute myocardial infarction, the authors reported that the plasma concentrations of various MMPs in patients with myocardial infarction were significantly increased [8].

They concluded MMP-9 may play a prominent physiological and pathological role during the stage of myocardial infarction to heart failure, more especially considering the fact that improper vascular redisposition of MMP-9 may promote atherosclerosis via weakening of atherosclerotic plaques [34]. It seems the potential mechanisms for reducing exercise-induced MMP-9 levels during cardiac rehabilitation is the changes in the levels of monocyte chemoattractant protein-1 (MCP-1) [35]. MCP-1 attracts monocytes to inflammatory sites located in the vascular subendothelial space. These monocytes are able to differentiate into macrophages and be converted to foam cells via absorbing oxidized low-density lipoproteins (Ox-LDL), suggesting an important role for MCP-1 in the pathogenesis of atherosclerosis [36]. The inhibition of MCP1 by relevant inhibitors prevents plaque inflammation and stops the rupture of disposed plaques [37]. On a better note, MCP-1 in myocytes and smooth vascular cells stimulates the expression of MMP enzymes and consequently induces inflammatory cytokines and MMP enzymes in cardiac myocytes [38].
Although MCP-1 levels were not measured in the present study, several studies have reported a reduction in MCP-1 following exercise suggesting a beneficial effect of physical exercise on heart patients. Thus, MCP-1 reduction is likely links to decreased plasma MMP-9 levels following regular exercise [39]. The activity of MMP inhibitors and the ratio of MMPs to TIMPs are as important as the secretion of tissue MMPs. Under normal conditions, a physiological balance between MMPs and TIMPs is stablished, and the extracellular matrix breakdown and synthesis is well-adjusted. Any disease or mechanical stress that results in decreased immune cells, increased inflammation, secretion of proinflammatory cytokines, and the activity of MMPs, consequently triggers the immediate inhibitory response particularly activation of TIMPs [40]. Hence, the inhibition of MMP proteolytic activity has been suggested as a therapeutic approach in various heart diseases [41-43].

\section{Conclusion}

In the present study, an inverse relationship between the bioactivity of MMP-9 and TIMP-1 was observed following eight weeks of exercise intervention in ischemic cardiac patients. Considering the favorable effects of physical exercise on cardiac rehabilitation, it can be suggested that patients with cardiac ischemia can accelerate their recovery process and reduce the risk of stroke reoccurrence by doing moderate intensity of physical exercise at least three time per week.

\section{Acknowledgement}

The authors wish to thank the participants in this research project.

\section{Funding}

This research did not receive any specific grant or funding from agencies in the public, commercial, or not-for-profit sectors.

\section{Conflict of Interest}

No conflicts of interest are declared by the authors.

\section{References}

1. Chughtai H, Ratner D, Pozo M, Crouchman JA, Niedz B, et al. (2011) Prehospital Delay and its Impact on Time to Treatment in ST-elevation Myocardial Infarction. Am J Emerg Med 29(4): 396-400.

2. Kim JH, Kim DJ, Jang HC, Choi SH (2011) Epidemiology of Micro- and Macrovascular Complications of Type 2 Diabetes in Korea. Diabetes Metab J 35(6): 571-577.

3. Lusis AJ (2000) Atherosclerosis. Nature 407(6801): 233-241.

4. Malekpour Dehkordi Z, Javadi E, Doosti M, Paknejad M, Nourbakhsh M, et al. (2011) The Effect of Alcoholic Garlic (Allium sativum) Extract on ABCA1 Expression in Human THP-1 Macrophages. Tehran Univ Med J 69(3): 146-152.

5. Fariba F, Esna Ashari F, Moradi M (2015) Comparison of Risk Factors Frequency in Patients with ST-elevation MI \& Patients with non STelevation MI in Hamadan City. Scientific Journal of Hamadan University of Medical Sciences 22(1): 11-15. 
6. Weiss TW, Furenes EB, Trøseid M, Solheim S, Hjerkinn EM, et al. (2010) Prediction of Cardiovascular Events by Matrix Metalloproteinase (MMP)-9 in Elderly Men. Thromb Haemost 103(3): 679-681.

7. Kjaer M (2004) Role of Extracellular Matrix in Adaptation of Tendon and Skeletal Muscle to Mechanical Loading. Physiol Rev 84(2): 649-698.

8. Tabatabaei Panah A, Akbarzadeh R, Khodaii Z, Ghaderian S (2016) Role of Matrix Metalloproteinase 1, 2, 3 and 9 in Acute Myocardial Infarction. Scientific-Research Journal of Shahed University 23(122): 29-39.

9. Cleutjens JP, Kandala JC, Guarda E, Guntaka RV, Weber KT (1995) Regulation of Collagen Degradation in the Rat Myocardium after Infarction. J Mol Cell Cardiol 27(6): 1281-1292.

10. Romanic AM, Burns Kurtis CL, Gout B, Berrebi Bertrand I, Ohlstein EH (2001) Matrix Metalloproteinase Expression in Cardiac Myocytes Following Myocardial Infarction in the Rabbit, Life Sciences 68(7): 799814.

11. Lee Johnson J (2014) Matrix Metalloproteinases and Their Inhibitors in Cardiovascular Pathologies: Current Knowledge and Clinical Potential. Metalloproteinases in Medicine 1: 21-36.

12. Arpino V, Brock M, Gill SE (2015) The Role of TIMPs in Regulation of Extracellular Matrix Proteolysis. Matrix Biol 44-46: 247-254.

13. Farshidi H (2004) Causes of Readmission in Ischemic Heart Disease Patients. Scientific information database 8(2): 67-71.

14. Antam ME, Selwyn AP, Loscalszo (2012) Ischemic heart disease. In: Lango DL, (Edi.). Harrisons principles of medicine. (18 ${ }^{\text {th }}$ Edn.). New York: McGrow Hill, Health Profession Division, pp. 1998-2015.

15. Dalir Z, VahdatFeizabadi E, Mazlom S, RajaeeKhorasani A (2013) The Effect of Short-term Cardiac Rehabilitation Program on Anxiety and Depression in Patients After Coronary Artery Bypass Surgery. Evidence Based Care Journal 3(8): 33-42.

16. Piepoli MF, Davos C, Francis DP, Coats AJ (2004) Exercise Training MetaAnalysis of Trials in Patients with Chronic Heart Failure (ExTraMATCH). BMJ 328(7433): 189.

17. Dakei SZ, Hemmat Far A, Azizbeigi K (2014) Effect of Resistance and Endurance Training Protocols on Functional Capacity and Quality of Life in Male Patients after Myocardial Infarction. Cardiovascular Nursing Journal 3(1): 26-33.

18. Volaklis K, Spassis A, Tokmakidis S, Komotini (2007) Land Versus Water Exercise in Patients with Coronary Artery Disease: Effects on Body Composition, Blood Lipids, and Physical Fitness. American Heart Journal 154(3): 560-566.

19. Afzalaghaiee E, Hadian M, Attarbashi Moghadam B, Tavakol K, Zandparsa A, et al. (2010) The Effects of Cardiac Rehabilitation on Changes in The Lipid Profile of Iranian Male and Female with Coronary Artery Disease Refered to Emam Khomeini Hospital Complex. MRJ 4(1-2): 45-49.

20. Hajighasemi A, Ravasi A, Kordi M, Rashidlamir A, Ghorghi A (2017) Investigation of the Effect of Cardiac Rehabilitation Program on Peripheral Blood Mononuclear Cells ABCA1 Gene Expression in Myocardial Infractions Patient. Journal of Knowledge \& Health 11(4): 23-29.

21. Shokrallahnia Roshan A, Sadeghi H, Shirani SH, Nejatian M (2013) Effects of Strength Training and Cardiac Rehabilitation Programs on the Biomechanical Parameters of Blood Flow Velocity and Blood Flow Rate and its Relation with Arterial Stiffness Index in Brachial and Femoral Arteries with Coronary Artery Bypass Grafting Patients (CABG). Journal of Rehabilitation 14(2): 38-45.

22. Hoang A, Tefft C, Duffy SJ, Formosa M, Henstridge DC, et al. (2008) ABCA1 Expression in Humans is Associated with Physical Activity and Alcohol Consumption. Atherosclerosis 197(1): 197-203.
23. Mostafavi S, Saeidi M, Heidari H (2012) Effects of a Comprehensive Cardiac Rehabilitation Program on the Quality of Life of Patients with Cardio-Vascular Diseases. Journal of Research in Rehabilitation Sciences 7(4): 516-524.

24. Borg GA (1962) Physical performance and perceived exertion: Gleerup Lund.

25. Pollock ML, Franklin BA, Balady GJ, Chaitman BL, Fleg JL, et al. (2000) Resistance Exercise in Individuals with and without Cardiovascular Disease Benefits, Rationale, Safety, and Prescription an Advisory from the Committee on Exercise, Rehabilitation, and Prevention, Council on Clinical Cardiology, American Heart Association. Circulation 101(7): 828-833.

26. Seidanloo F, Farzanegi P (2015) Changes in Matrix Metallo-proteinases 2, 9 and Tissue Inhibitor of Matrix Metalloproteinase 1 to Synchronized Exercise Training and Celery, as an Herbal Supplement, in Overweight Women. Modaress Journal of Medical Sciences: Pathobiology 18(1): 107-118.

27. Kadoglou NP, Moustardas P, Kapelouzou A, Katsimpoulas M, Giagini A, et al. (2013) The Anti-Inflammatory Effects of Exercise Training Promote Atherosclerotic Plaque Stabilization in Apolipoprotein E Knockout Mice with Diabetic Atherosclerosis. Eur J Histochem 57(1): e3.

28. Koskinen SO, Heinemeier KM, Olesen JL, Langberg H, Kjaer M (2004) Physical Exercise Can Influence Local Levels of Matrix Metalloproteinases and their Inhibitors in Tendon -Related Connective Tissue. J Appl Physiol 96(3): 861-864.

29. Mackey AL, Donnelly AE, Swanton A, Murray F, Turpeenniemi Hujanen T (2006) The Effects of Impact and Non-Impact Exercise on Circulating Markers of Collagen Remodelling in Humans. J Sports Sci 24(8): 843848.

30. Hoier B, Nordsborg N, Andersen S, Jensen L, Nybo L, et al. (2012) Proand Anti-Angiogenic Factors in Human Skeletal Muscle in Response to Acute Exercise and Training. J Physiol 590(3): 595-606.

31. Kim HE, Dalal SS, Young E, Legato MJ, Weisfeldt ML, et al. (2000) Disruption of the Myocardial Extracellular Matrix Leads to Cardiac Dysfunction. The Journal of Clinical Investigation 106(7): 857-866.

32. Murphy G, Cockett MI, Stephens PE, Smith BJ, Docherty AJ (1987) Stromelysin is an Activator of Procollagenase. A Study with Natural and Recombinant Enzymes. Biochemistry Journal 248(1): 265-268.

33. Lee SW, Song KE, Shin DS, Ahn SM, Ha ES, et al. (2005) Alterations in peripheral Blood Levels of TIMP-1, MMP-2, and MMP-9 in Patients with Type-2 Diabetes. Diabetes Res Clin Pract 69(2): 175-179.

34. Galis ZS, Khatri JJ (2002) Matrix Metalloproteinases in Vascular Remodeling and Atherogenesis: the Good, the Bad, and the Ugly. Circ Res $90(3): 251-262$.

35. Farzanegi P (2014) Impact of the Synchronization of Portulaca Oleracea and Aerobic Training on Levels of MMP2 and MMP9 and TIMP1 in Diabetic Women Type II. Res Mol Med 2(2): 34-39.

36. Lin J, Kakkar V, Lu X (2014) Impact of MCP-1 in Atherosclerosis. Curr Pharm Des 20(28): 4580-4588.

37. Niu J, Kolattukudy PE (2009) Role of MCP-1 in Cardiovascular Disease: Molecular Mechanisms and Clinical Implications. Clin Sci 117(3): 95109.

38. Azfer A, Niu J, Rogers LM, Adamski FM, Kolattukudy PE (2006) Activation of Endoplasmic Reticulum Stress Response During the Development of Ischemic Heart Disease. Am J Physiol Heart Circ Physiol 291(3): H14111420.

39. Kazemi A (2017) Effect of High Intensity Interval Training on Visceral and Subcutaneous Levels of MCP-1 and Plasma Insulin and Glucose in Male Rats. Razi Journal of Medical Sciences 23(152): 29-37. 
40. Dastani M, Rashidlamir A, Alizadeh A, Seyedolhosseini M, Ebrahimi AA (2014) Effects of 8 Weeks of Aerobic Exercise on Matrix Metalloproteinase-9 and Tissue Inhibitor Levels in Type II Diabetic Women. Zahedan Journal of research in medical Sciences 16(6): 12-15.

41. Woessner JF (1991) Matrix Metalloproteinases and their Inhibitors in Connective Tissue Remodeling. FASEB Journal 5(8): 2145-2154.

42. Spinale FG, Coker ML, Krombach SR, Mukherjee R, Hallak H, et al. (1999) Matrix Metalloproteinase Inhibition during the Development of Congestive Heart Failure: Effects on Left Ventricular Dimensions and Function. Circulation Research 85(4): 364-376.

ISSN: 2574-1241

DOI: 10.26717/BJSTR.2021.38.006172

Mostafa Dastani. Biomed J Sci \& Tech Res

(c) (P) This work is licensed under Creative

Submission Link: https://biomedres.us/submit-manuscript.php
43. Haq M, Shafii A, Zervos EE, Rosemurgy AS (2000) Addition of Matrix Metalloproteinase Inhibition to Conventional Cytotoxic Therapy Reduces Tumor Implantation and Prolongs Survival in a Murine Model of Human Pancreatic Cancer. Cancer Research 60(12): 3207-3211.

$\begin{array}{ll}\text { BIOMEDICAL } & \text { Assets of Publishing with us } \\ \text { RESEARCHES } & \text { - Global archiving of articles } \\ & \text { - Immediate, unrestricted online access } \\ & \text { - Rigorous Peer Review Process } \\ & \text { - Authors Retain Copyrights }\end{array}$

